\title{
1 Throwing performance in water polo is related to in-water shoulder 2 proprioception
}

Water polo players require a high level of upper-extremity strength, flexibility and coordination to achieve a peak level of throwing performance. Increased levels of shoulder proprioceptive acuity, strength and range of motion (ROM) have been previously associated with higher sporting performance. A coachrating scale, used to quantify an athlete's kicking proficiency in soccer; was adapted in the current study to measure each coach's subjective expert opinion regarding athletes' throwing mechanics, velocity, and accuracy. To examine this hypothesis shoulder proprioception acuity of 18 water polo players was measured both in-water and on-land using an AMEDA apparatus and correlated with coach-rated throwing performance and clinical measures of shoulder strength and ROM. There was a moderate positive correlation between the in-water and the on-land proprioception acuity $(r=0.47, p<$ $0.05)$. The in-water score showing a strong positive correlation with coach rated throwing mechanics $(r=0.68, p<0.05)$ and velocity $(r=0.75, p=0.02)$, suggesting that superior proprioception acuity contributed to fast, mechanically-efficient throwing. These findings support the notion that inwater proprioceptive acuity is an important determinant of the throwing performance achieved by water polo athletes and its measurement may be a valuable adjunct to current athlete screening. opinion 
27 The actions of overhead throwing, passing and swimming in water polo place

28 repeated loads on the shoulder complex (Miller, Evans, Adams, Waddington, \&

29 Witchalls, 2017). To excel at the sport, athletes require sufficient joint range of

30 motion (ROM), muscle strength, endurance, and stability (Riemann \& Lephart,

31 2002). Functional stability of the shoulder joint relies on the interaction of dynamic

32 (muscular) and static (capsulo-ligamentous, osseous, tendon) structures (Wilk, 1993),

33 mediated by the sensorimotor system (Riemann \& Lephart, 2002). Proprioception is

34 an afferent input of this sensorimotor system that is of increasing interest within sport

35 and rehabilitative fields (Ager et al., 2017). For example, it has been shown that a

36 decreased proprioception sense can increase risk of injury (Safran, Borsa, Lephart,

$37 \mathrm{Fu}, \&$ Warner, 2001), injury re-occurrence and contribute to poor recovery in

38 overhead athletes with shoulder pain (Fyhr, 2015).

39 The term proprioception has evolved to become an umbrella term which

40 includes the sub-modalities of joint position sense (JPS), sense of tension or

41 replication of force magnitudes (Dover \& Powers, 2003), kinaesthesia (Riemann,

42 Myers, \& Lephart, 2002), vibration detection (Clark et al., 2015) and changes in limb

43 velocity (Arzi, Krasovsky, Pritsch, \& Liebermann, 2014). The assessment of

44 conscious proprioception is divided into three sub-components: JPS, the sensation of

45 movement (kinaesthesia), and sense of tension (Lephart, Pincivero, Giraido, \& Fu,

46 1997). Kinaesthesia assesses the ability to not only detect movement but also the

47 direction, and is assessed by measuring threshold to detection of passive motion,

48 whilst JPS is assessed by measuring the accuracy of active or passive replication of

49 position. (Lephart, Pincivero, \& Rozzi, 1998; Riemann et al., 2002).

50 Central processing may play a role in sporting performance (Han,

51 Waddington, Adams, Anson, \& Liu, 2016). Indeed, proprioception is not just neural 
52 input. A recent systematic review demonstrated the role of proprioception in

53 developing a neuromuscular response to a stimulus, involving sensory input, central

54 processing and motor response in a closed loop (Witchalls, Blanch, Waddington, \&

55 Adams, 2012). Elite soccer players have been shown to dedicate less central

56 processing to proprioception but more attention to the technical aspects of the game

57 such as decision making and awareness of team positioning (Han, Anson,

58 Waddington, \& Adams, 2014). Whether this is due to an athlete's intensive training,

59 and subsequent neuromuscular adaption, or to inherent genetic factors, is unknown

60 (Han, Waddington, Anson, \& Adams, 2015).

61 The combination of proprioception, and accompanying neuromuscular

62 feedback mediated by the central nervous system (CNS), results in conscious

63 awareness of joint position and motion, and unconscious joint stabilisation through

64 spinal mediated reflexes (Lephart et al., 1998). Peripheral articular and

65 musculotendinous receptors provide afferent proprioceptive input to the CNS and

66 influence the reflex activity and joint stability during throwing activities (Myers \&

67 Lephart, 2002). It has been hypothesised that the increased shoulder ROM observed

68 in throwing athletes as an adaptive response may, over time, induce trauma to the

69 shoulder capsule (Mota \& Ribeiro, 2012). It is suggested that the increased ROM

70 may damage mechanoreceptors, consequently reducing afferent input and increasing

71 an athlete's throwing-related risk of injury (Mota \& Ribeiro, 2012; Nocera, Rubley,

72 Holcomb, \& Guadagnoli, 2006).

73 From a performance perspective, water polo athletes, like other throwing

74 athletes, have been found to have specific neuromuscular adaptations thought to be

75 important for skilled performance. For example, increased shoulder internal rotation

76 (IR) strength (Bloomfield, Blanksby, Ackland, \& Allison, 1990), and increased ER 
77 ROM in the dominant throwing shoulder (Witwer \& Sauers, 2006) are proposed to

78 enhance performance by increasing ball velocity while allowing a longer acceleration

79 phase (Whiteley, Adams, Ginn, \& Nicholson, 2010). Furthermore, proprioception

80 has also been associated with an athlete's level of attainment in sport (Han, Anson,

81 Waddington, Adams, \& Liu, 2015; Han, Waddington, et al., 2015). This suggests

82 that a higher level of proprioception acuity contributes to enhanced motor control of

83 joints, and thus superior athletic performance (Waddington \& Shepherd, 1996).

84 While proprioception acuity is unlikely to be the only determinant of throwing

85 success, the relationship between proprioception and successful performance in

86 water polo athletes warrants investigation (Whiteley, Adams, Nicholson, \& Ginn,

87 2008).

Despite the importance of optimal biomechanical and neuromuscular

89 characteristics, such as an athlete's strength, ROM and throwing mechanics, the

90 overall evaluation of a player's ability, and subsequent team selection, is often based

91 largely on the expert opinion of the team coaching staff (Calder \& Durbach, 2015).

92 There are a number of factors that influence this opinion, such as grading of

93 performance, anthropometry, tactical and psychological performance (Calder \&

94 Durbach, 2015; Nash \& Collins, 2006; Phillips, Davids, Renshaw, \& Portus, 2010).

95 Additionally, it appears that a coach's rating of skilled performance may be

96 correlated with an athlete's proprioceptive ability (Cameron \& Adams, 2003). For

97 example, in Australian Football, a positive correlation has been demonstrated

98 between coach-rated kicking performance and an athlete's level of proprioception

99 acuity measured using a purpose-built active movement extent discrimination

100 apparatus (AMEDA) to measure the swing leg JPS (Cameron \& Adams, 2003).

101 Collectively, these findings support the notion that proprioceptive acuity is an 
102 important determinant of the level of performance achieved by athletes and its

103 measurement may be a valuable adjunct to the current methodology of athletic

104 screening, due to its association with sporting ability.

105 To our knowledge, only one previous study has investigated shoulder

106 proprioception in water polo athletes, and this study focused on the relationship

107 between active joint repositioning error and shoulder strength in 20 male national

108 league athletes (Mota \& Ribeiro, 2012). The main findings were correlations

109 between shoulder joint position error at $30^{\circ}$ of external rotation (ER) and both

110 eccentric ER strength and concentric IR strength (Mota \& Ribeiro, 2012). The

111 authors suggested that athletes with reduced proprioception may be more prone to

112 throwing-related injury due to a delayed neuromuscular protective reflex and

113 insufficient shoulder strength to decelerate the arm in the follow-through phase of

114 throwing (Mota \& Ribeiro, 2012).

115 Given the potential importance of proprioception to both injury prevention

116 and successful performance (Fyhr, 2015), it is fundamental that proprioception is

117 measured accurately and reliably. In testing proprioception, it is important that the

118 tests maximise the similarity between the laboratory test and real-life function

119 (ecological validity) (Gibson, 1979). The AMEDA methodology aims to maximise

120 the ecological validity of proprioception testing through replicating activities similar

121 to the requirements of water polo (Han et al., 2016). The sport of water polo presents

122 athletes and coaches with a unique challenge of performing overhead throwing

123 activities within water rather than land as their base of support, requiring athletes to

124 simultaneously tread water whilst passing and shooting the ball. Recent evidence in

125 ankle proprioception suggests that dual task demands decrease an individual's

126 proprioception performance (Yasuda, Sato, Iimura, \& Iwata, 2014). To date, no 
127 previous study has measured shoulder proprioception of water polo athletes in their 128 aquatic playing environment.

129 The aims of the current study were to assess shoulder proprioception JPS,

130 both in-water and on-land, and to examine the relationship between proprioception,

131 shoulder strength, ROM, and coach-rated throwing performance. For the current

132 study, an AMDEDA apparatus (Whiteley et al., 2008) was adapted to enable

133 assessment of JPS during shoulder abduction and ER (throwing position), measured

134 whilst treading water. If JPS is important in throwing performance, then a

135 relationship between proprioception and throwing performance is expected.

136 Moreover, it is also anticipated that the coach's expert opinion/rating of a player's

137 skill will be positively correlated with the athlete's proprioceptive acuity level. This

138 research has implications for coaches, as proprioception training may refine neural

139 programs, allowing athletes to execute movement faster due to less conscious effort

140 being required (Han et al., 2016). Furthermore, proprioception testing may have a

141 role in talent identification and an athlete's likely skill acquisition within water polo. 
143 Methods

144 Participants

145 Twelve male and six female players were recruited from a state level water polo

146 training squad (see supplementary material 1 for athlete demographics). Initial

147 contact was made via an email from the high-performance manager detailing the

148 proposed study. Potential participants were required to first fill out a questionnaire

149 containing demographic information, and to check for eligibility. To be eligible for

150 inclusion, athletes were required to have more than five years' playing experience

151 and be in water polo training a minimum of three days/week consistently over the

152 previous year. As a team, athletes were attending up to three strength sessions/week

153 and four or five pool sessions. An athlete's competition level was determined by

154 their highest playing level in the preceding 12 months (club, state, national level).

155 Athletes were excluded if they had shoulder pain or injury that prevented them from

156 participating in full unmodified team training, had experienced a severe injury, such

157 as shoulder subluxation/dislocation or surgery, in the preceding 12 months, or had

158 shoulder pain when adopting the required test position of shoulder abduction and ER.

159 Ethical approval and informed consent were obtained prior to commencement

160 of the study. The study was approved by the the University of Canberra Human

161 Research Ethics Committee (HREC 16-47) and all athletes were invited to provide

162 informed consent before being included in the study. Athletes were familiarised with

163 the AMEDA device and were tested during their team's training session 48-hours

164 after their last training session. All measurements were taken from the dominant

165 shoulder, defined as the player's preferred throwing/shooting arm. 
167 Shoulder JPS was assessed with a purpose-built shoulder AMEDA shown in Figure

168 1. The AMEDA was configured for active proprioception testing (Naughton, Adams,

169 \& Maher, 2002; Whiteley, Ginn, Nicholson, \& Adams, 2009) and adapted so that it

170 could be used to assess athletes both in-water and on-land. The apparatus consisted

171 of a contact plate attached to the end of a hand-driven shaft used to set the stop point

172 for the hand when moving backwards with the elbow flexed to $90^{\circ}$ and shoulder

173 externally rotated to $75^{\circ}$. The apparatus was mounted on either the pool edge (for

174 pool testing) or wooden boxes (for on-land testing) so that the height could be

175 individualised to ensure the dorsum of the hand contacted the middle of the contact

176 plate/disc (position one). The five stop settings (1-5) used were $2.0 \mathrm{~cm}$ apart, and

177 were located backwards of stop 1, thus progressively increasing the ER as the

178 distance withdrawn was increased (Figure 2). A height adjustable padded plate was

179 positioned at the lumbar-thoracic junction to ensure athletes remained centred with

180 respect to the device.

181 All male players were tested without a shirt, in their team allocated briefs,

182 and the females were tested in their team bathing suit. For the on-land testing,

183 athletes stood with their feet shoulder width apart, head facing straight ahead, eyes

184 fixed on a target on a wall $15 \mathrm{~m}$ in front of them, so that they had no direct vision of

185 the hand or target plate. For the in-water testing, athletes were required to tread

186 water, eyes focussed on a target on the opposite side of the pool, $15 \mathrm{~m}$ in front of

187 them. Proprioception was tested using both the in-water and the on-land AMEDA,

188 for the dominant throwing arm only. Total testing time was 40 minutes (20 minutes

189 per device) with 20 minutes rest between each apparatus. The order of the on-water

190 or on-land testing was randomised. 
The AMEDA tests were conducted using active movements and all athletes

192 were given a standardised briefing and were familiarised with the test protocol.

193 Athletes were informed that there were five displacement distances, numbered 1

194 (smallest), 2, 3, 4, and 5 (largest), in increasing order of ER extent. Athletes were

195 then familiarised by performing three rounds on the device, moving from smallest to

196 largest distance, and making contact at each of the five stops (15 stops in total).

197 Athletes then undertook 50 trials without feedback, wherein all five stops

198 were presented 10 times in a random order. For each trial, the device was moved to

199 the stop position; the athlete then moved their arm from the starting position (hand

200 held at water level or hand held in front of the body in the same position as the pool)

201 and externally rotated their shoulder to touch the stop plate. Athletes then identified

202 the position as either position $1,2,3,4$ or 5 by using their memory of the extent of

203 movement from the familiarisation series. The reported stop value was recorded, but

204 no feedback was given as to accuracy of the response. For consistency, the same

205 evaluator was used for each participant. 
207 Measurement of shoulder strength and ROM was conducted in the week prior to data

208 collection as part of the annual pre-season screening for the water polo program.

209 Neither the team physiotherapist nor the athletes were blinded to the ROM and

210 strength results. All athletes were measured by the same team physiotherapist, to

211 reduce protocol variation error. Before testing, athletes performed a 5-minute water

212 polo-specific warm up, and were familiarised with the testing procedures.

213 Passive shoulder IR and ER ROM were measured with the athlete lying

214 supine with their shoulder in $90^{\circ}$ abduction, $90^{\circ}$ elbow flexion and the forearm in

215 neutral (zero position). The shoulder was stabilised anteriorly by the physiotherapist

216 and no scapular movement was permitted. For IR, an inclinometer (TruMedical

217 Baseline ${ }^{\circledR}$ bubble $360^{\circ}$ ) was positioned centrally on the dorsal surface of the

218 forearm, $2 \mathrm{~cm}$ proximal to the styloid process of the ulna. The testing position was

219 reproduced for ER on the ventral aspect (Kevern, Beecher, \& Rao, 2014). Maximal

220 isometric shoulder IR and ER strength were measured using a hand-held

221 dynamometer (HHD; JTech Medical, Powertrack II Commander). Athletes were

222 required to sit on a plinth, with hips and knees at $90^{\circ}$ and feet flat on the floor. The

223 arm was positioned so the shoulder was at $0^{\circ}$ abduction, elbow flexed to $90^{\circ}$ with the

224 forearm in a neutral position. For IR, the dynamometer was centred on the volar

225 aspect of the distal forearm, $2 \mathrm{~cm}$ proximal to the radial styloid, and on the dorsal

226 aspect for testing ER. Athletes completed three maximal 5s isometric tests for both

227 IR and ER, which the physiotherapist met with equal and opposite force. A 5s rest

228 was given between the three tests and if a breaking contraction occurred, the reading

229 was omitted before executing the test again (Dollings, Sandford, O'Conaire, \& 
230 Lewis, 2012). Three measurements were performed and the average taken. All

231 measurements were recorded in kilograms force (kgf).

232

233 Coach rating of throwing performance

234 Each coach, from the male and the female team, was surveyed to obtain their expert

235 opinion of each athlete's throwing skill. Coaches were asked to rate throwing

236 performance on a 1-10 scale. Ratings were obtained for each athlete regarding their

237 throwing accuracy, velocity, and mechanics. Athletes were also rated for overall

238 strength and flexibility. Coaches were instructed to record the overall impression of

239 each athlete's throwing, strength, and flexibility based on their observation of

240 training and competition. Please see the supplementary information for the full

241 coaches' survey.

242 
244 For the in-water and on-land AMEDA testing, the perceived stop position was

245 tabulated against actual position in a stimulus-response matrix (Cameron \& Adams,

246 2003). For both the in-water and on-land device, ability to discriminate between

247 adjacent angles (1-2, 2-3, 3-4 and 4-5) was determined by calculating the area

248 under the curve (AUC) of the receiver operating characteristics (ROC), with a curve

249 therefore generated for each paired comparison (Witchalls, Waddington, Adams, \&

250 Blanch, 2013). The mean AUC score was then calculated from the four pair-wise

251 AUCs. This score gives the athletes' overall proprioceptive ability for shoulder

252 movements into ER (Witchalls, Newman, Waddington, Adams, \& Blanch, 2012). An

253 AUC score of 0.50 is equivalent to chance performance and a score of 1.0 represents

254 perfect shoulder joint position discrimination.

255 The relationship between the AUC scores for the in-water and on-land

256 AMEDAs was investigated using Pearson product moment correlation coefficient

257 and r-values of $0.1,0.3$ and 0.5 were interpreted as small, medium and large

258 respectively (Cohen, 1992). A paired t-test was conducted on the AUC scores to

259 establish if there was any difference between the scores obtained in the two test

260 environments. A Bland-Altman plot was used to assess whether any differences were

261 consistent across the full range of scores.

262 To determine their relationship to shoulder proprioception in a throwing

263 position, the AMEDA AUC scores were also correlated with coach rated throwing

264 performance and athletes' measured shoulder IR strength, ER strength, and ROM.

265 All statistical analysis was performed using SPSS version 23.0 (SPSS Inc., Chicago,

266 IL, USA) with the level of significance set at $\mathrm{p}<0.05$. 


\section{Results}

269 The mean proprioception score for in-water testing was $0.68 \pm 0.06$ and $0.71 \pm 0.08$

270 for on-land testing. No significant difference in mean scores between the two

271 environments $(\mathrm{p}=0.10)$ was found, suggesting that the absence of a firm base of

272 support did not impair shoulder proprioception in these water-polo players. The

273 mean of the differences between scores for the two devices was $0.06 \pm 0.08$ (limits of

274 agreement, -0.03 to 0.15 ) and there was an even spread of difference scores around

275 the zero point on a Bland Altman plot (Figure 3).

276 A moderate positive correlation between the in-water and the on-land

277 AMEDA $(\mathrm{r}=0.47, \mathrm{p}<0.05)$ was found. The in-water AMEDA score showed a

278 strong positive correlation with coach rated throwing mechanics $(r=0.68, p<0.05)$

279 and velocity $(r=0.75, p=0.02)$.

280 Athletes' number of sport-specific training years was not correlated with

281 proprioception acuity score for either in-water $(r=-0.03, p=0.91)$ or on-land testing

$282(\mathrm{r}=-0.41, \mathrm{p}=0.09)$. However an athlete's highest playing level in the last 12 months

283 was strongly correlated with in-water proprioception acuity $(\mathrm{r}=0.59, \mathrm{p}=0.01)$.

284 Coach-rated strength was significantly correlated with the measured IR

285 strength obtained from manual testing $(r=0.89, \mathrm{p}<0.01)$. Coach-rated throwing

286 mechanics was significantly related to ER ROM $(r=0.72, p=0.04)$ ER strength $(r=$

$2870.86, p=0.04)$ and coach rated throwing velocity $(r=0.91, p<0.01)$. For these

288 players, coach-rated throwing accuracy was significantly positively correlated with

289 their IR strength $(r=0.88, p=0.02)$ and coach rating of strength $(r=0.76, p<0.05)$

290 and was negatively correlated with coach-rated flexibility $(r=-0.76, p<0.05)$.

291 Players who the coach perceived to be more flexible were seen to have worse scores

292 for perceived accuracy. See Table 1 for all correlations. 
293

$294<$ Insert Table 1>

295 


\section{Discussion}

297 Main findings: The primary aim of the current study was to determine whether the 298 proprioception underpinning throwing was environment-specific, by correlating 299 scores obtained from an adapted in-water AMEDA device with the scores obtained

300 with an on-land device. A further aim was to better understand the relationship

301 between the variables: proprioception in a throwing posture, strength, and ROM, and

302 expert coaches' ratings of aspects of throwing performance. Water polo athletes

303 commonly exhibit sport-specific adaptive changes in their mobility, strength, and

304 proprioception (Miller et al., 2017), and many teams collect routine athlete screening

305 information in order to provide an indication of an athlete's overall and sport specific

306 capabilities. The current study confirmed the face validity of coach ratings as seen in

307 their relationship with proprioception scores. The in-water AMEDA proprioceptive

308 acuity scores demonstrated a strong positive correlation with coach-rated throwing

309 mechanics $(r=0.68, p<0.05)$ and velocity $(r=0.75, p=0.02)$ whereas the on-land

310 scores did not correlate with coach ratings. These findings suggest that good in-water

311 proprioception contributed to fast, mechanically-efficient throwing and gives further

312 weight to the importance of employing ecologically valid methodology when

313 assessing proprioception. Additionally, coach rated athletic strength was also

314 significantly correlated with athletes' clinical measures of IR strength $(r=0.89, p<$

3150.01 ), suggesting that manifestations of strength in defence and attack are visible to

316 coaches in this highly physical sport.

317 Development of the in-water AMEDA: Compared with passive tests and 318 supine tests, active-movement based measures of shoulder proprioception more 319 closely resemble normal function (Naughton et al., 2002), and thus have greater 320 ecological validity when assessing proprioception acuity in throwing athletes 
321 (Whiteley et al., 2008). To assess shoulder proprioception actively, researchers have

322 previously measured JPS of overhead points using arm flexion (Naughton et al.,

323 2002) and ER (Whiteley et al., 2008) in standing participants. Overhead position JPS

324 with shoulder flexion demonstrated the importance of consistent preparation in

325 movement. What is unknown, however, is the relevance with a sporting population

326 (Naughton et al., 2002). The game of water polo requires athletes to perform

327 overhead throwing activities with water as the base of support. Thus, for the current

328 study, ecological validity (Han, Anson, Waddington, \& Adams, 2013) was

329 maximised by modifying the AMEDA apparatus (Whiteley et al., 2008) to enable

330 testing in water. To reduce proprioceptive feedback and encourage athletes to

331 perform abduction/ER using their preferred movement pattern, arm movements were

332 completed without elbow support, as fixation may alter JPS (Tripp, 2006).

333 The role of the AMEDA in talent identification: One of the findings of the

334 current study was that there was a moderate positive correlation between in-water

335 and on-land proprioception test scores. Although athletes who scored well on the

336 proprioception test on-land also scored well on the in-water test, the water testing

337 procedure was seen as superior to the on-land testing due to its association with

338 coach-rated water polo throwing performance, and playing level achieved in the

339 previous 12-months, and thus it may better reflect skilled performance. These

340 findings are consistent with a previous study demonstrating that superior

341 proprioception acuity to be significantly associated with level of performance

342 achieved by athletes (Han, Anson, et al., 2015), and gives weight to the hypothesis

343 that proprioception testing may have a role in talent identification and an athlete's

344 likely skill acquisition. Future AMEDA studies in water polo are warranted across

345 multiple joints to investigate the relationship between proprioception acuity, training 
346 years and level of sporting attainment, and could play a role in identifying talented 347 athletes.

348 Shoulder ROM adaptation and AMEDA testing: When comparing

349 performance of the dominant to the non-dominant shoulder, water polo athletes

350 consistently demonstrated adaptive change and increased dominant shoulder ER

351 ROM (Elliott, 1993; Witwer \& Sauers, 2006). Explanations as to why overhead

352 athletes have a gain in dominant ER ROM have included osseous adaption (retro-

353 torsion) of the humerus from both genetic and overhead activity (Whiteley, Ginn, et

354 al., 2009) and accumulative micro-trauma to the soft tissue structures of the shoulder

355 from throwing activity (Witwer \& Sauers, 2006). Gain in dominant shoulder ER

356 ROM has been associated with increased throwing performance level in baseball

357 players (Whiteley et al., 2010) and reduced injury risk (Wilk et al., 2015). Athletes'

358 throwing proficiency is improved due to increased ER ROM that is available in the

359 cocking action before strain is transferred to shoulder tissues (Whiteley, Adams,

360 Nicholson, \& Ginn, 2009) and also gives a longer available acceleration phase

361 (Whiteley et al., 2010). In the current study, coach-rated throwing mechanics were

362 significantly correlated with clinical measures of athletes' ER ROM, and ER ROM

363 correlated with on-land proprioception but not in-water proprioceptive acuity. The

364 current findings are consistent with those from a previous AMEDA study on baseball

365 players, where it was found that non-dominant humeral retro-torsion and active

366 proprioception were positively and significantly correlated (Whiteley et al., 2008).

367 Further, with one outlier removed, the dominant shoulder retro-torsion scores

368 demonstrated a similar pattern and proprioception was moderately correlated with

369 retro-torsion (Whiteley et al., 2008). 
371 that the five predefined angles employed in the proprioception test cover a section of

372 the relevant range but are not adjusted for individual athletes' ER ROM. JPS has

373 been shown to be affected by the required range of shoulder elevation angle in

374 unconstrained tasks (Suprak, Osternig, van Donkelaar, \& Karduna, 2006). In active

375 positioning tasks, afferent input may be different for athletes with larger ER (closer

376 to their mid-range) than athletes with less ER (closer toward their end ROM), due to

377 the muscle spindle playing a greater role in mid-range elevation and capsular-

378 ligamentous receptors being maximally stimulated toward the end of range (Lephart

379 et al., 1997; Safran et al., 2001; Whiteley et al., 2008). It has been proposed that

380 identification of players at risk of shoulder injury, due to sub-clinical injury, could be

381 achieved by targeting players with low proprioception/mean proprioception score,

382 despite sufficient ER ROM (Whiteley et al., 2008). Although study of prospective

383 shoulder injury was beyond the scope of the current study, given the association of

384 athletes' ROM to both performance and proprioception acuity, further research

385 investigating the relationship between ROM, proprioception, and injury in water polo 386 is warranted.

387 Since the use of the AMEDA is a new application of this test, it was not

388 possible to do an a priori power calculation. However, the values for variance within

389 this population, established by this study, can be used to calculate power for future

390 studies. The levels of significance seen in the current study indicate that the test is

391 able to discriminate performance differences in relatively small populations.

392 Shoulder strength and AMEDA testing: Findings from a previous study

393 suggested that water polo players with reduced shoulder strength have reduced

394 proprioception (Mota \& Ribeiro, 2012). In contrast, the current study found no 
395 association between measures of athletes' IR or ER strength and in-water or on-land

396 mean proprioception score. A possible reason for the difference in these findings

397 could be the different methodology employed, with Mota \& Robeiro., (2012) using a

398 passive positioning and active reproduction protocol to assess angle reproduction

399 error (difference between actual and target at $30^{\circ} \mathrm{ER}$ ) compared to the AUC score

400 obtained from JPS of five positions, as used in the current study. Whilst passive

401 positioning of the limb improves the reliability of testing, the clinical applicability to

402 real life function remains questionable (Ager et al., 2017). Moreover, athletes in the

403 current study were completing three strength sessions per week on top of the

404 plyometric training from pool throwing. Closed chain strength training has been

405 demonstrated to improve both function and proprioception in a short time frame

406 (Myers \& Oyama, 2009), and 6 weeks of plyometric training improves

407 proprioception (Swanik et al., 2002). Potentially the team strength training program

408 may have confounded the results with regards to strength. Although, in the current

409 study, strength and proprioception were not correlated, the in-water proprioception

410 score and coach rated throwing performance were. A larger sample would provide a

411 more robust analysis of the association between proprioception and strength. Given

412 the known relationship between strength, performance, and proprioception, further

413 investigation regarding the current AMEDA protocol and targeted plyometric and

414 closed chain strengthening is recommended to determine if the in-water AMEDA is

415 sensitive to short term change. Potential exists for use of the AMEDA as pre-season

416 screening instrument as well as for obtaining return-to-sport baseline post injury. 


\section{Conclusion}

418 The in-water AMEDA device score had a strong and positive correlation with coach

419 rated throwing speed and mechanics whereas results obtained from the on-land

420 AMEDA did not. The overall evaluation of a water polo player's ability, and

421 subsequent team selection, is often based largely on the expert opinion of the team

422 coaching staff. These findings support the notion that in-water proprioceptive acuity

423 is an important determinant of an athlete's throwing performance and its

424 measurement may be a valuable adjunct for coaches regarding current athlete

425 screening and future talent identification. Although beyond the scope of the current

426 study, reduced proprioception acuity has been proposed to increase the risk of injury

427 amongst water polo players. Further investigation regarding the in-water

428 proprioception device and humeral retro-torsion through indirect ultrasound

429 measurement with prospective injury follow up is warranted to explore this

430 hypothesis. This method would allow exploration of the association between physical

431 structure of the shoulder, shoulder proprioception and shoulder injury in water polo,

432 to evaluate the impact of achieving the shoulder angles that are used within water

433 polo throwing. Moreover, once the role of proprioception in shoulder injury is

434 established, plyometric training and proprioception development/retraining of water

435 polo players should be investigated. 
436 Acknowledgements: The research team would like to thank the support of the

437 Queensland Academy of Sport's Sport Performance Innovation and Knowledge

438 Excellence unit for their assistance with this project. Thank you also to Mrs Alice

439 Hyslop for her assistance with data collection and recruitment.

440

441 Funding statement: We received financial support through a joint Queensland

442 Academy of Sport-University of Canberra PhD scholarship to Ms Andrea Hams.

443 Grant number 2014000296

444

445 Declaration of interest: The authors have no financial affiliation or involvement with

446 any commercial organisation that has a direct financial interest in any matter

447 included in this manuscript.

448 
450 Ager, A., Roy, J., Roos, M., Belley, A., Cools, A., \& Hébert, L. (2017). Shoulder proprioception: How is it measured and is it reliable? A systematic review.

453 Arzi, H., Krasovsky, T., Pritsch, M., \& Liebermann, D. G. (2014). Movement control in patients with shoulder instability: a comparison between patients after open

Calder, J., \& Durbach, I. (2015). Decision support for evaluating player performance

in rugby union. Int J Sports Sci Coach, 10(1), 21-37. doi:10.1260/1747-

Bloomfield, J., Blanksby, B., Ackland, T., \& Allison, G. (1990). The influence of strength training on overhead throwing velocity of elite water polo players. Aust J Sci Med Sport, 63-67. 9541.10 .1 .21

Cameron, M., \& Adams, R. (2003). Kicking footedness and movement discrimination by elite australian rules footballers. J Sci Med Sport, 6(3), 266274. doi:10.1016/S1440-2440(03)80020-8

Clark, N. C., Röijezon, U., Treleaven, J., Institutionen för, h., Luleå tekniska, u., \& Hälsa och, r. (2015). Proprioception in musculoskeletal rehabilitation. Part 2: Clinical assessment and intervention. Manual therapy, 20(3), 378-387.

472 Dollings, H., Sandford, F., O’Conaire, E., \& Lewis, J. (2012). Shoulder strength 473 testing: the intra- and inter-tester reliability of routine clinical tests, using the 
PowerTrack $^{\mathrm{TM}}$ II Commander. Shoulder Elbow, 4(2), 131-140. doi:10.1111/j.1758-5740.2011.00162.x

476 Dover, G., \& Powers, M. (2003). Reliability of joint position sense and forcereproduction measures during internal and external rotation of the shoulder. $J$ Athl Train, 38(4), 304-310.

479 Elliott, J. (1993). Shoulder pain and flexibility in elite water polo players. Physiotherapy, 79(10), 693-697. doi:10.1016/S0031-9406(10)60004-1

481 Fyhr, C. (2015). The effects of shoulder injury on kinaesthesia: A systematic review and meta-analysis. Man Ther, 20(1), 28-37.

483 Gibson, J. (1979). The ecological approach to visual perception. Hillsdale: NJ:

$484 \quad$ Erlbaum.

485 Han, J., Anson, J., Waddington, G., \& Adams, R. (2013). Proprioceptive performance of bilateral upper and lower limb joints: side-general and sitespecific effects. Exp Brain Res, 226(3), 313-323. doi:10.1007/s00221-013$3437-0$

489 Han, J., Anson, J., Waddington, G., \& Adams, R. (2014). Sport attainment and proprioception. Int J Sports Sci Coach, 9(1), 159-170.

491 Han, J., Anson, J., Waddington, G., Adams, R., \& Liu, Y. (2015). The role of ankle proprioception for balance control in relation to sports performance and injury. Biomed Res Int, 2015, 1-8. doi:10.1155/2015/842804

494 Han, J., Waddington, G., Adams, R., Anson, J., \& Liu, Y. (2016). Assessing proprioception : A critical review of methods. J Sport Health Sci, 5(1), 8090. doi:10.1016/j.jshs.2014.10.004 
497 Han, J., Waddington, G., Anson, J., \& Adams, R. (2015). Level of competitive success achieved by elite athletes and multi-joint proprioceptive ability. $J \mathrm{Sci}$ Med Sport, 18(1), 77-81. doi:http://dx.doi.org/10.1016/j.jsams.2013.11.013

500 Kevern, M., Beecher, M., \& Rao, S. (2014). Reliability of measurement of glenohumeral internal rotation, external rotation, and total arc of motion in 3 test positions. J Athl Train, 49(5), 640-646. doi:10.4085/1062-6050-49.3.31

Lephart, S., Pincivero, D., Giraido, J., \& Fu, F. H. (1997). The role of proprioception in the management and rehabilitation of athletic injuries. Am J Sports Med, 25(1), 130-137. doi:10.1177/036354659702500126

Lephart, S., Pincivero, D., \& Rozzi, S. (1998). Proprioception of the ankle and knee. Sports Medicine, 25(3), 149-155. doi:10.2165/00007256-199825030-00002

Miller, A., Evans, K., Adams, R., Waddington, G., \& Witchalls, J. (2017). Shoulder injury in water polo: A systematic review of incidence and intrinsic risk

514 Myers, J., \& Lephart, S. (2002). Sensorimotor deficits contributing to glenohumeral factors. J Sci Med Sport, 21(4), 368-377. doi:10.1016/j.jsams.2017.08.015

Mota, N., \& Ribeiro, F. (2012). Association between shoulder proprioception and instability. Clin Orthop Relat Res, 400(400), 98-104. doi:10.1097/00003086-

517 Myers, J., \& Oyama, S. (2009). Sensorimotor training for shoulder injury. Athl Train, 1(5), 199-208. doi:10.3928/19425864-20090611-01

519 Nash, C., \& Collins, D. (2006). Tacit knowledge in expert coaching: Science or art? 520 Quest, 58(4), 465-477. doi:10.1080/00336297.2006.10491894 
521 Naughton, J., Adams, R., \& Maher, C. (2002). Discriminating overhead points of contact after arm raising. Percept Mot Skills, 95(3_suppl), 1187-1195.

524 Nocera, J., Rubley, M., Holcomb, W., \& Guadagnoli, M. (2006). The effects of 525 repetitive throwing on shoulder proprioception and internal and external 526 rotation strength. J Sport Rehabil, 15(4), 351-362.

527 Phillips, E., Davids, K., Renshaw, I., \& Portus, M. (2010). Expert performance in sport and the dynamics of talent development. Sports Medicine, 40(4), 271-

530 Riemann, B., \& Lephart, S. (2002). The sensorimotor system, part I: The physiologic basis of functional joint stability. J Athl Train, 37(1), 71-79.

532 Riemann, B., Myers, J., \& Lephart, S. (2002). Sensorimotor system measurement techniques. J Athl Train, 37(1), 85-98.

534 Safran, M., Borsa, P., Lephart, S., Fu, F., \& Warner, J. (2001). Shoulder proprioception in baseball pitchers. J Shoulder Elbow Surg, 10(5), 438-444.

536 Suprak, D., Osternig, L., van Donkelaar, P., \& Karduna, A. (2006). Shoulder joint position sense improves with elevation angle in a novel, unconstrained task. $J$ Orthop Res, 24(3), 559-568. doi:10.1002/jor.20095

539 Swanik, K. A., Swanik, C. B., Lephart, S. M., Lephart, S. P., Stone, D. A., \& Fu, F. 540 H. (2002). The effects of shoulder plyometric training on proprioception and selected muscle performance characteristics. J Shoulder Elbow Surg, 11(6),

543 Tripp, B. (2006). Functional multijoint position reproduction acuity in overheadthrowing athletes. J Athl Train, 41(2), 146-153. 
545 Waddington, G., \& Shepherd, R. (1996). Ankle injury in sports: role of motor control systems and implications for prevention and rehabilitation. Phys Ther Rev, 1(2), 79-87. doi:10.1179/ptr.1996.1.2.79

548 Whiteley, R., Adams, R., Ginn, K., \& Nicholson, L. (2010). Playing level achieved, 549 throwing history, and humeral torsion in Masters baseball players. $J$ Sports Sci, 28(11), 1223-1232.

Whiteley, R., Adams, R., Nicholson, L., \& Ginn, K. (2008). Shoulder proprioception is associated with humeral torsion in adolescent baseball players. Phys Ther Sport, 9(4), 177-184. doi:http://dx.doi.org/10.1016/j.ptsp.2008.07.002

554 Whiteley, R., Adams, R., Nicholson, L., \& Ginn, K. (2009). Reduced humeral torsion predicts throwing-related injury in adolescent baseballers. $J$ Sci Med Sport, 13(4), 392-396. doi:10.1016/j.jsams.2009.06.001

Whiteley, R., Ginn, K., Nicholson, L., \& Adams, R. (2009). Sports participation and humeral torsion. J Orthop Sports Phys Ther, 39(4), 256-263.

Wilk, K. (1993). The strength characteristics of internal and external rotator muscles in professional baseball pitchers. Am J Sports Med, 21(1), 61.

Wilk, K., Macrina, L., Fleisig, G., Aune, K., Porterfield, R., Harker, P., . . Andrews, J. (2015). Deficits in glenohumeral passive range of motion increase risk of shoulder injury in professional baseball pitchers. Am J Sports Med, 43(10), 2379-2385. doi:doi:10.1177/0363546515594380

Witchalls, J., Blanch, P., Waddington, G., \& Adams, R. (2012). Intrinsic functional deficits associated with increased risk of ankle injuries: A Systematic review with meta-analysis. Br J Sports Med, 46(7), 515-523. doi:10.1136/bjsports2011-090137 
569 Witchalls, J., Newman, P., Waddington, G., Adams, R., \& Blanch, P. (2012). Functional performance deficits associated with ligamentous instability at the ankle. J Sci Med Sport, 16(2), 89-93. doi:10.1016/j.jsams.2012.05.018

572 Witchalls, J., Waddington, G., Adams, R., \& Blanch, P. (2013). Chronic ankle instability affects learning rate during repeated proprioception testing. Phys Ther Sport, 15(2), 106-111. doi:10.1016/j.ptsp.2013.04.002

575 Witwer, A., \& Sauers, E. (2006). Clinical measures of shoulder mobility in college water-polo players. J Sport Rehabil, 15(1), 45-57. doi:10.1123/jsr.15.1.45

577 Yasuda, K., Sato, Y., Iimura, N., \& Iwata, H. (2014). Allocation of attentional 578 resources toward a secondary cognitive task leads to compromised ankle 579 proprioceptive performance in healthy young adults. Rehabil Res Pract, 580 2014, 170304-170304. doi:10.1155/2014/170304 
Table 1: Correlation of on-land and in-water proprioception, measured shoulder internal rotation (IR) and external rotation (ER) strength (ST) and range of motion (ROM) with coach rated performance in water polo. Reported as r-value (p value).

\begin{tabular}{|c|c|c|c|c|c|c|c|c|c|c|}
\hline & & \multicolumn{5}{|c|}{ Coach rated performance } & \multicolumn{4}{|c|}{ Athlete screening } \\
\hline & \multirow{2}{*}{$\begin{array}{c}\text { AMEDA } \\
\text { Land }\end{array}$} & \multicolumn{3}{|c|}{ Throwing } & \multicolumn{2}{|c|}{ Characteristics } & \multicolumn{2}{|c|}{ Strength } & \multicolumn{2}{|c|}{ Range of motion } \\
\hline & & Accuracy & Velocity & Mechanics & Strength & Flexibility & IR & ER & IR & ER \\
\hline Water & $0.47 *(0.05)$ & $0.30(0.51)$ & $0.75^{*}(0.02)$ & $0.68^{*}(0.05)$ & $0.30(0.44)$ & $-0.16(0.69)$ & $0.16(0.56)$ & $0.07(0.79)$ & $-0.35(0.19)$ & $0.33(0.21)$ \\
\hline Land & & $0.25(0.60)$ & $0.33(0.39)$ & $0.52(0.15)$ & $0.19(0.63)$ & $0.58(0.10)$ & $0.13(0.63)$ & $0.21(0.43)$ & $0.06(0.82)$ & $0.70 *(<0.01))$ \\
\hline Accuracy & & & $0.36(0.43)$ & $0.41(0.36)$ & $0.76^{*}(0.05)$ & $-0.76 *(0.05)$ & $0.88 *(0.02)$ & $0.29(0.57)$ & $-0.16(0.77)$ & $0.07(0.90)$ \\
\hline Velocity & & & & $0.91 *(<0.01)$ & $0.47(0.21)$ & $-0.14(0.72)$ & $0.31(0.41)$ & $0.64(0.09)$ & $-0.10(0.81)$ & $0.55(0.16)$ \\
\hline Mechanics & & & & & $0.52(0.15)$ & $-0.06(0.87)$ & $0.55(0.15)$ & $0.86^{*}(<0.01)$ & $-0.10(0.81)$ & $0.72 *(0.04)$ \\
\hline Strength & & & & & & $-0.32(0.40)$ & $0.89 *(<0.01)$ & $0.70(0.06)$ & $-0.10(0.81)$ & $-0.013(0.98)$ \\
\hline Flexibility & & & & & & & $-0.28(0.50)$ & $0.27(0.51)$ & $0.02(0.97)$ & $0.55(0.16)$ \\
\hline IR ST & & & & & & & & $0.57 *(0.02)$ & $-0.43(0.10)$ & $0.01(0.96)$ \\
\hline ER ST & & & & & & & & & $-0.32(0.23)$ & $0.29(0.28)$ \\
\hline IR ROM & & & & & & & & & & $-0.60(0.82)$ \\
\hline
\end{tabular}


$585 \quad$ Figure 1

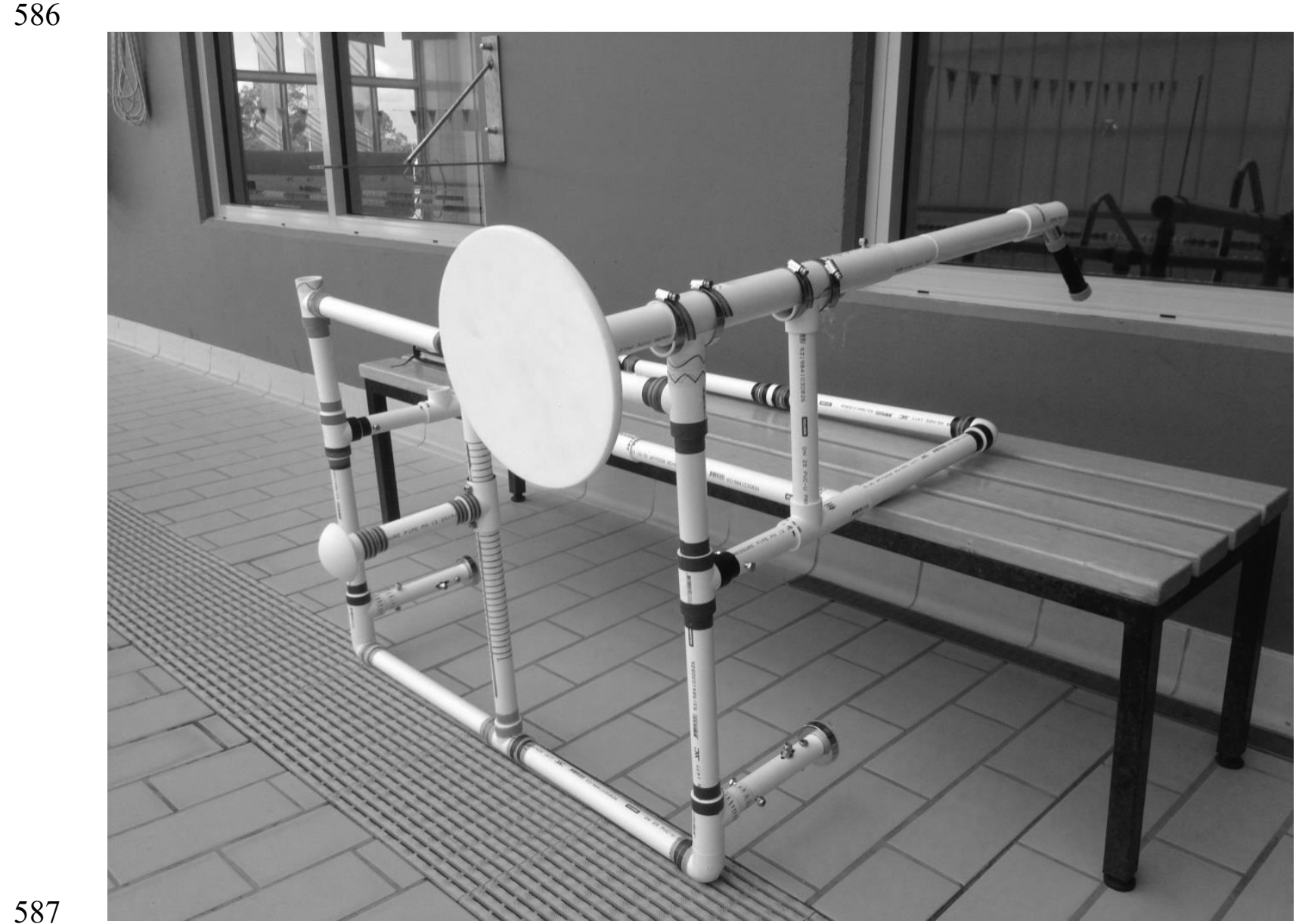


589 Figure 2

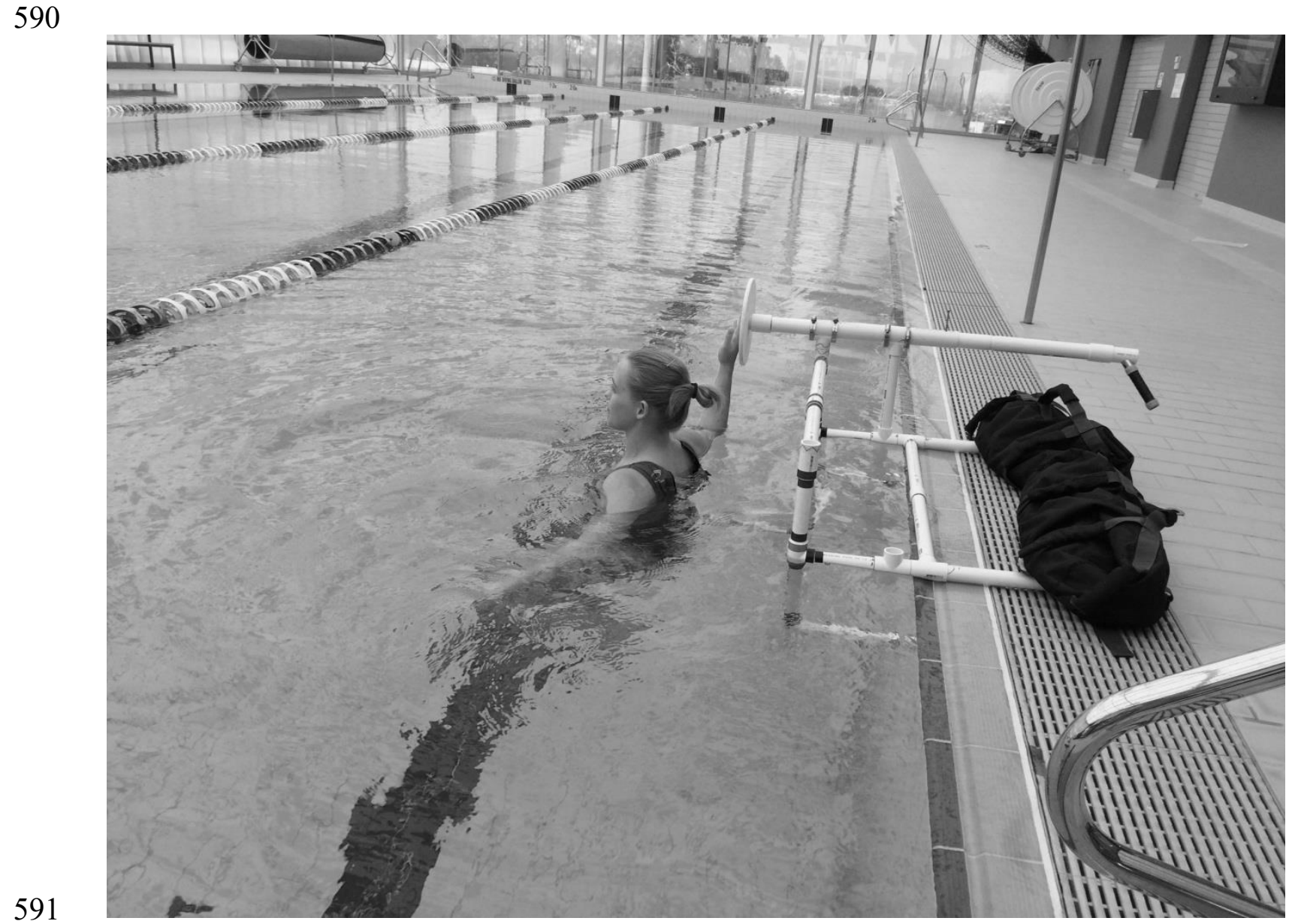


Bland Altman plot of on-land and in-water proprioception of the dominant shoulder

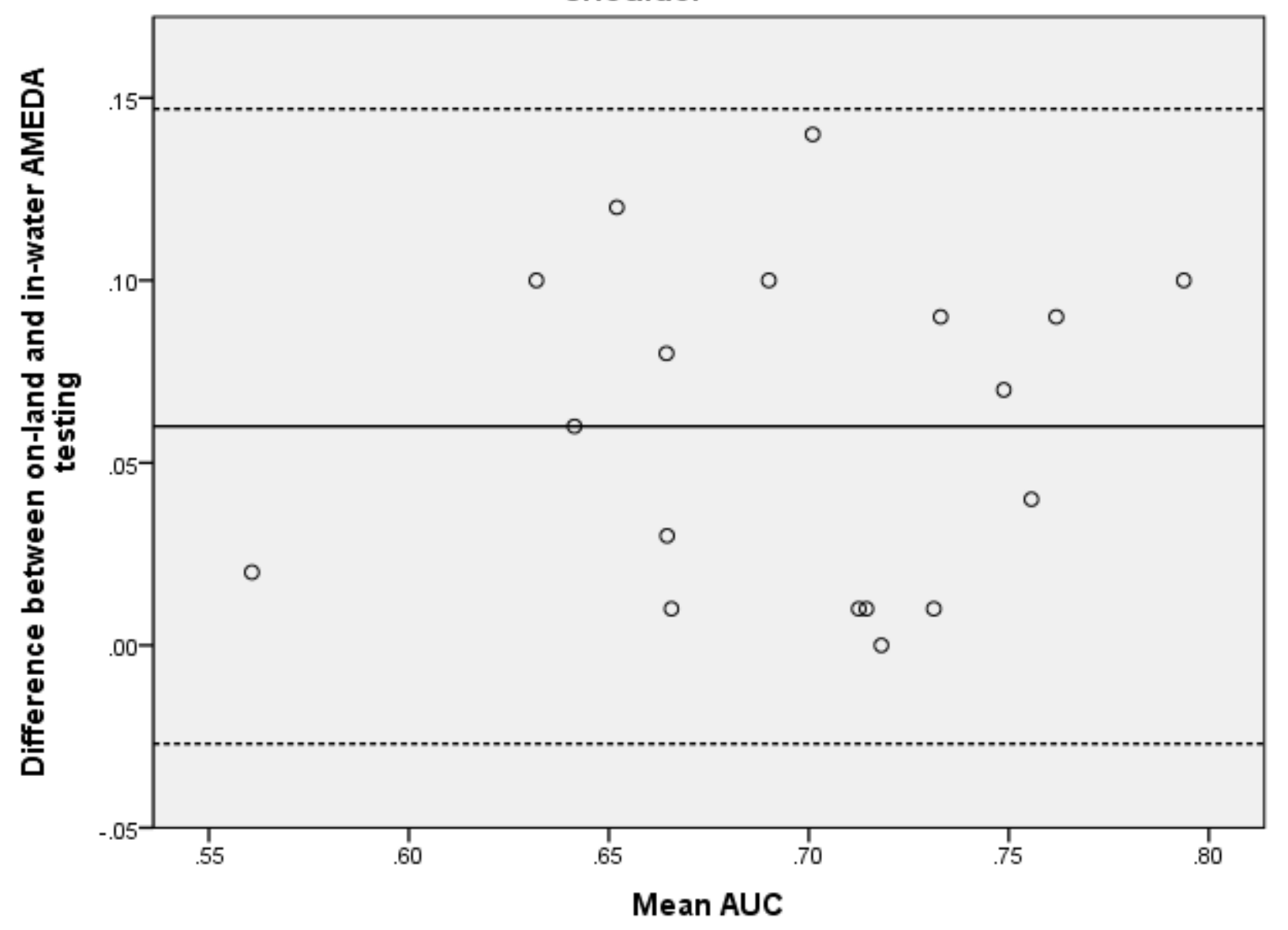

593

594

595 
596 Figure captions

597 Figure 1: The purpose built shoulder AMEDA device that can be adapted to both in598 water and on-land testing

599 Figure 2: The AMEDA apparatus mounted on either the pool edge to ensure the

600 dorsum of the hand contacted the middle of the contact plate. Position five of the five 601 (1-5) stop settings is demonstrated, as the athlete abducts and ER the shoulder.

602 Figure 3: Bland Altman plot of on-land and in-water proprioception of the dominant 603 shoulder, -- upper and lower level of agreement (LOA). AUC - area under the curve 604 (proprioception acuity). 